\title{
Nutrition risk and items contributing to nutrition risk in naturally occurring retirement communities in Ontario, Canada
}

\author{
C. Mills ${ }^{1}$ and C. Donnelly ${ }^{1}$ \\ ${ }^{1}$ School of Rehabilitation Therapy, Faculty of Health Sciences, Queen's University, Kingston, Canada
}

Older adults are the fastest-growing demographic group in Canada ${ }^{(1)}$. Most older adults want to age-in-place within their communities $^{(2)}$. One-third of these community-dwelling older adults in Canada are at nutrition risk, the risk of poor dietary intake and nutritional status, with consequences including increased frailty, decreased quality of life, increased hospitalization, and higher mortality rates $^{(3)}$. It is therefore important to understand what contributes to nutrition risk in this population in order to effectively address these contributing factors. The objectives of this study were to determine levels of nutrition risk among residents of seven naturally occurring retirement communities (NORCs) in the province of Ontario, Canada, and to determine which items contributed the most to nutrition risk in these residents.

Participants were recruited from NORCs in the cities of Kingston, Belleville, Hamilton, and London, in the province of Ontario, Canada. Individuals were screened for nutrition risk using Seniors in the Community: Risk Evaluation for Eating \& Nutrition 14 (SCREEN-14), a valid and reliable tool for screening for nutrition risk in community-dwelling older adults 4 consisting of 14 questions. Scores can range from 0 to 64 , with scores less than 54 indicating nutrition risk ${ }^{(4)}$. A score $\leq 2$ for an individual response item indicates nutrition risk for that item ${ }^{(4)}$. The percentage of individuals at nutrition risk was calculated, as were the percentage of individuals at risk for each response item in the tool.

In total, 127 individuals were screened. SCREEN-14 scores ranged from 19 to 62 , with a mean score of $45(\mathrm{SD}=7.90)$. The percentage of individuals at risk for each item on the SCREEN-14 tool follows: weight change in past six months: $34.7 \%$; not trying to change weight in past six months: 4.9\%; perception of weight (more/less than it should be): $66.4 \%$; skipping meals: $42.2 \%$; limiting or avoiding certain foods: $40.6 \%$; poor appetite: $16.4 \%$, inadequate servings of fruit and vegetables: $57.8 \%$, meat and alternatives: 39.1 milk products: $58.3 \%$; inadequate fluid intake: $35.9 \%$; swallowing problems: $21.1 \%$; chewing problems: $25.8 \%$; use of commercial meal replacements: $28.9 \%$; eating alone: $57.8 \%$; problems getting groceries: $25.2 \%$; difficulties with meal preparation or dissatisfaction with meals prepared by others: $41.3 \%$.

There were high levels of nutrition risk in these NORC residents, with $87.4 \%$ of individuals at nutrition risk. For many individual item scores, a significant percentage of the older adults who were screened were at risk. Items contributing the most to nutrition risk were perception of weight, eating alone, inadequate intake of fruit and vegetables, inadequate intake of milk products, skipping meals, difficulties with meal preparation or dissatisfaction with meals prepared by others, and limiting or avoiding certain foods. In order to improve nutrition risk in this population these risk factors need to be addressed.

\section{Acknowledgements}

We would like to thank the residents of the naturally occurring retirement communities who participated in our study.

\section{References}

1. Statistics Canada (2018) Population Projections for Canada [Available at: https://www150.statcan.gc.ca/n1/pub/91-520-x/91-520-x2019001-eng.htm].

2. NIA (2019) Enabling the Future Provision of Long-Term Care in Canada [Available at: https://www.nia-ryerson.ca/s/Enabling-the-FutureProvision-of-Long-Term-Care-in-Canada-5ye6.pdf].

3. Ramage-Morin PL, Gilmour H \& Rotermann M (2017) Health Reports 28(9), 17-27.

4. Keller HH, Goy R \& Kane SL (2005) Eur J Clin Nutr 59(10), 1149-57. 